\title{
Analysis of SMOS sea surface salinity data using DINEOF
}

\author{
Aida Alvera-Azcárate ${ }^{\mathrm{a}, *}$, Alexander Barth ${ }^{\mathrm{a}, \mathrm{b}}$, Gaëlle Parard ${ }^{\mathrm{a}}$, Jean-Marie Beckers ${ }^{\mathrm{a}}$ \\ a AGO-GHER-MARE, University of Liège, Allée du Six Aout, 17, Sart Tilman, Liège 4000, Belgium \\ b F.R.S.-FNRS (National Fund for Scientific Research), Brussels, Belgium
}

\section{A R T I C L E I N F O}

\section{Article history:}

Received 31 July 2015

Received in revised form 23 December 2015

Accepted 18 February 2016

Available online 28 February 2016

\begin{abstract}
A B S T R A C T
An analysis of daily Sea Surface Salinity (SSS) at $0.15^{\circ} \times 0.15^{\circ}$ spatial resolution from the Soil Moisture and Ocean Salinity (SMOS) satellite mission using DINEOF (Data Interpolating Empirical Orthogonal Functions) is presented. DINEOF allows reconstructing missing data using a truncated EOF basis, while reducing the amount of noise and errors in geophysical datasets. This work represents a first application of DINEOF to SMOS SSS. Results show that a reduction of the error and the amount of noise is obtained in the DINEOF SSS data compared to the initial SMOS SSS data. Errors associated to the edge of the swath are detected in 2 EOFs and effectively removed from the final data, avoiding removing the data at the edges of the swath in the initial dataset. The final dataset presents a centered root mean square error of 0.2 in open waters when comparing with thermosalinograph data at their original spatial and temporal resolution. Constant biases present near land masses, large scale biases and latitudinal biases cannot be corrected with DINEOF because persistent signals are retained in high order EOFs, and therefore these need to be corrected separately. The signature of the Douro and Gironde rivers is detected in the DINEOF SSS. The minimum SSS observed in the Gironde plume corresponds to a flood event in June 2013, and the shape and size of the Douro river shows a good agreement with chlorophyll-a satellite data. These examples show the capacity of DINEOF to remove noise and provide a full SSS dataset at a high temporal and spatial resolution with reduced error, and the possibility to retrieve physical signals in zones with high initial errors.
\end{abstract}

(c) 2016 Elsevier Inc. All rights reserved.

\section{Introduction}

Sea Surface Salinity (SSS) is being measured globally by the Soil Moisture and Ocean Salinity (SMOS) satellite mission, allowing obtaining an unprecedented spatial and temporal coverage in the measurement of this variable. SMOS measures radio frequency emission in the first centimeters of the upper ocean. The SMOS salinity mission has a target accuracy of $\sim 0.2$ over $100 \mathrm{~km}^{2}$ and 30 days (Lagerloef \& Font, 2010). Salinity is derived through the relation between brightness temperature (BT), sea surface temperature (SST) and sea roughness. This relation is more reliable for high values of BT and SST, so the precision of the salinity estimates decreases at high latitudes. Other sources of error for remotely-sensed SSS are sea roughness, astronomical radiation sources (e.g. galactic glint and cosmic background), sun glint, atmosphere attenuation of the emitted signal and proximity to land and ice (Lagerloef, Schmitt, Schanze, \& Kao, 2010; Boutin et al., 2012). In addition, illegal anthropic radio emissions (radio-frequency interference, $\mathrm{RFI}$ ) in the protected L-band frequency result in permanently or intermittently contaminated zones of the ocean, such as the European seas and Asia (Boutin et al., 2012). These sources of error result in noise and biases that need to be corrected, and gaps in the satellite SSS fields.

\footnotetext{
* Corresponding author.

E-mail address: a.alvera@ulg.ac.be (A. Alvera-Azcárate).
}

Several features have been analyzed using SMOS SSS data, showing the importance of this dataset. For example, the North Atlantic subtropical salinity maximum (Kolodziejczyk, Hernandez, Boutin, \& Reverdin, 2015), the Gulf Stream meanders (Reul et al., 2014), and the Amazon river plume (Grodsky, Reverdin, Carton, \& Coles, 2014; Reul et al., 2014; Fournier, Chapron, Salisbury, Vandemark, \& Reul, 2015). Processes at smaller scales, or features near land masses are more difficult to analyze using SMOS SSS because of the systematic errors due to the proximity of land.

DINEOF (Data Interpolating Empirical Orthogonal Functions) is a technique to reconstruct missing data in geophysical datasets that uses a truncated EOF basis in an iterative approach. DINEOF has been successfully applied to sea surface temperature (e.g. Alvera-Azcárate, Barth, Rixen, \& Beckers, 2005), chlorophyll-a and winds (e.g. AlveraAzcárate, Barth, Beckers, \& Weisberg, 2007), and total suspended matter (e.g. Sirjacobs et al., 2011; Alvera-Azcárate, Vanhellemont, Ruddick, Barth, \& Beckers, 2015). This work represents the first attempt at using DINEOF with salinity data. The challenges faced in the use of DINEOF to analyze SSS are the high level of noise in the SMOS SSS data, the presence of constant biases and errors due for example to the presence of land masses, and the lack of knowledge about the spatial and temporal signatures in SSS and how to separate them from the mentioned errors. Constant biases cannot be corrected with DINEOF so these should be removed separately, using for example the approach suggested by Kolodziejczyk et al. (2016-in this issue). 
The aim of this work is to assess if DINEOF is able to detect and remove noise and errors from the SMOS SSS dataset at the highest temporal and spatial resolution provided by the data. By removing noise and errors at the highest resolution, these data do not enter into the computation of composites and can provide therefore improved estimates at 10-day or monthly averages.

This work is organized as follows: the satellite and in situ data used are described in Section 2. Section 3 describes the methods used in this work: pre-processing of the data, and a description of DINEOF and the outlier detection methodology. The results section consists of a sensitivity study, a comparison with independent data, an assessment of the spatial resolution achieved by the data, the analysis of the final dataset and the description of the EOF basis used for the computation of the final dataset. These are presented in Section 4. We conclude this work in Section 5.

\section{Data used}

\subsection{Satellite data}

Level 2 Ocean Salinity User Data Product (UDP) version 5.50, provided by ESA, are used in this study. The domain covered is the North-East Atlantic Ocean and Mediterranean Sea, as shown in Fig. 1. Daily data for 2013 consisting of the ascending orbits have been selected, and the SSS data calculated using Roughness Model 1 (Yin, Boutin, Martin, \& Spurgeon, 2012) have been retained for this study. Only ascending orbits are used to avoid working with data that have a different accuracy, as the descending orbits present larger errors than the ascending orbits. The data are kept at the original spatial resolution, which is of $0.15^{\circ} \times 0.15^{\circ}$. Data located farther than $\pm 300 \mathrm{~km}$ of the centre of the track are subject to higher errors (Zine et al., 2008; Yin et al., 2014), and are therefore typically removed (i.e. Boutin et al., 2014). In this work, however, we have retained the full swath in order to assess if these errors at the edges of the swath can be discarded through the DINEOF analysis. Mediterranean Sea data are also subject to large errors, but the data are retained as well in this work in order to assess if DINEOF is able to extract a physically meaningful signal from the initial SSS.
For a qualitative assessment of the salinity data near the river plume of the Douro river we use 8-day composites of MODIS (Moderate Resolution Imaging Spectroradiometer) Aqua level 3 chlorophyll-a data, with a spatial resolution of $4 \mathrm{~km}$ and obtained from http://oceancolor. gsfc.nasa.gov/.

\subsection{In situ data}

In situ salinity data for 2013 have been extracted from the World Ocean Database (WOD) 2013 (Boyer et al., 2013), http://www.nodc. noaa.gov/and the Coriolis Data Centre (http://www.coriolis.eu.org/). Data from all platforms are extracted (Argo, CTD, drifting buoys and moored buoys), and the shallowest value is retained for each profile (with a maximum depth of $2 \mathrm{~m}$ ). Note that most Argo data stop measuring when reaching depths shallower than $5 \mathrm{~m}$, therefore most of the data used here have an effective depth that is deeper than $2 \mathrm{~m}$. The in situ data have been randomly distributed into a training dataset $(90 \%$ of the data) and a validation dataset (10\% of the data). The training dataset has been used to compute a monthly climatology using divand (n-dimensional Data Interpolating Variational Analysis, Barth et al. (2014)). This technique is equivalent to DIVA (Troupin et al., 2012) but multi-dimensional analyses can be handled, which allows taking into account for example the temporal correlation existing in the data. A SSS field has been calculated for each month of 2013, with a spatial resolution of 0.5 degrees. The spatial correlation length has been established to 10 degrees for the first guess and 1 degree for the short-scale background. The temporal correlation length is 90 days for the first guess and 15 days for the short-scale background. Signal-tonoise ratio is set up to 20 . The spatial and temporal correlation scales have been optimized by minimizing the RMS between the analysis and a cross-validation dataset consisting of $5 \%$ of the initial data chosen at random. Signal-to-noise ratio was optimized by the same procedure. These monthly fields, interpolated to daily values, are removed from the SMOS SSS fields prior to the DINEOF analyses in order to work with anomalies, and an example for February, July and December is shown in Fig. 2. Evidently, the presence of features like the Gulf Stream or the subtropical salinity maximum in the background fields depends

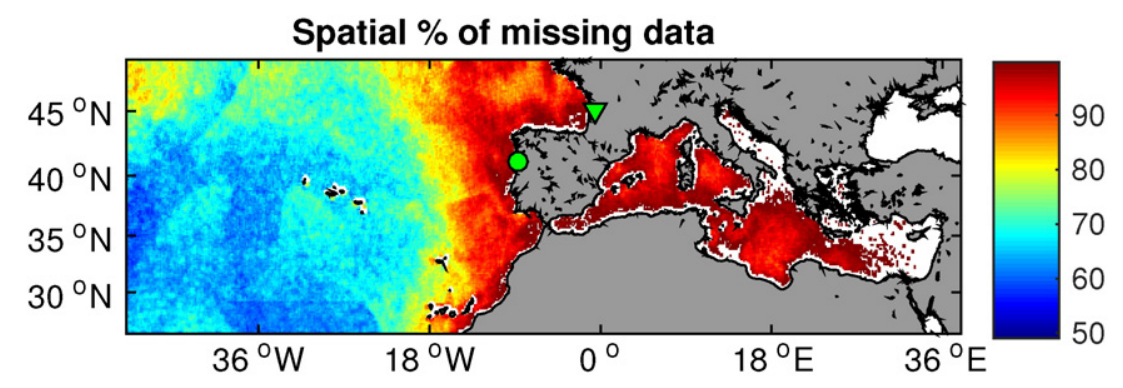

Temporal $\%$ of missing data

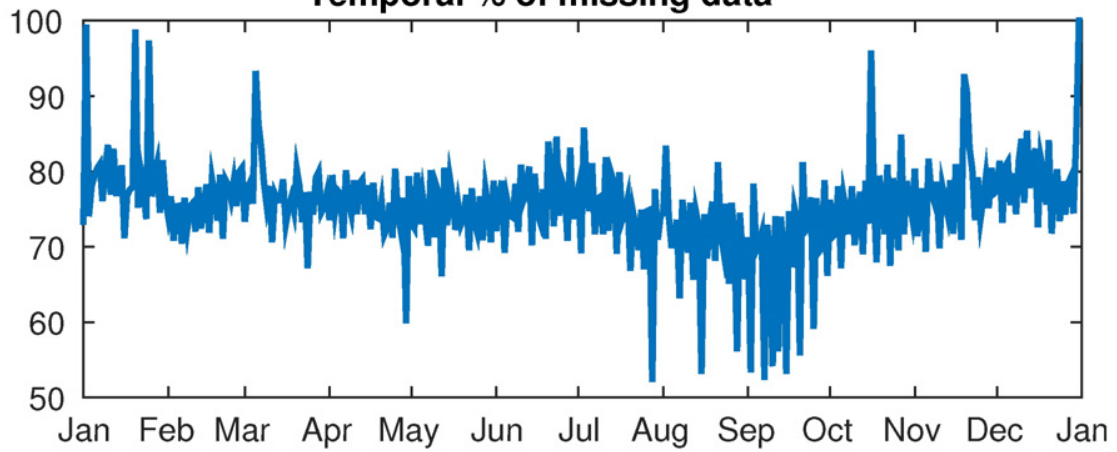

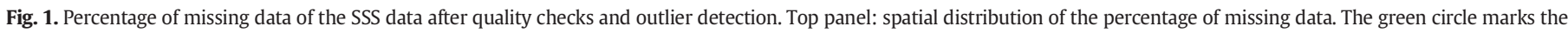
position of the Douro river mouth, and the red triangle marks the position of the Gironde estuary. Bottom panel: temporal evolution of the percentage of missing data. 
Background SSS February 2013

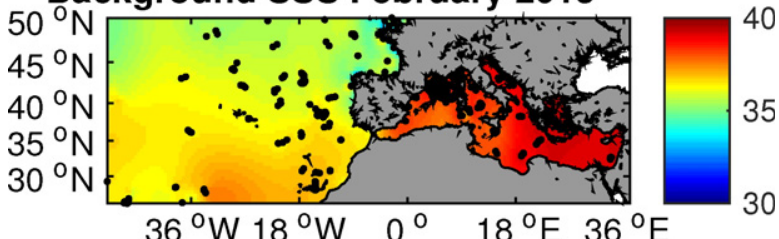

Background SSS July 2013

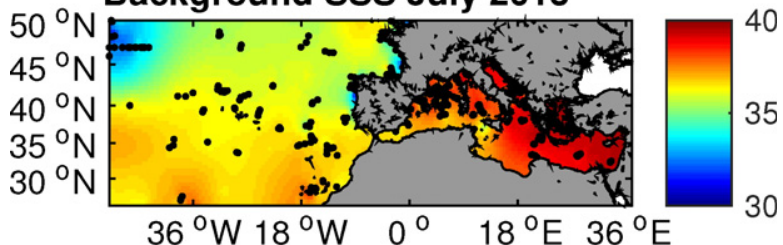

Background SSS December 2013

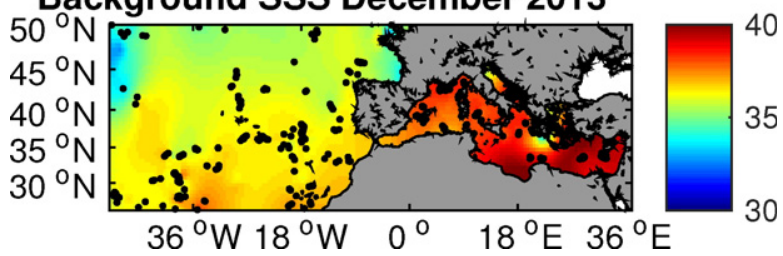

Fig. 2. Example of monthly background SSS fields calculated using in situ data. Top panel: in situ SSS for February 2013; middle panel: in situ SSS for July 2013; bottom panel: in situ SSS for December 2013.

strongly on the presence of enough data to resolve them, as in the case of the Gulf Stream weaker amplitude in February (top panel of Fig. 2). The validation dataset (10\% of the initial data, not used in the background) is used in the comparison with the original SSS and DINEOF reconstruction, in Section 4.

Thermosalinograph (TSG) data from Voluntary Observing Ships distributed by Legos http://www.legos.obs-mip.fr are also used in this work for comparison purposes. The TSG SSS data are measured every 5 min over a series of fixed tracks along the North Atlantic Ocean and the Mediterranean Sea.

\section{Methods}

\subsection{Data pre-processing}

Several quality flags, provided with the original data, are used in order to remove data suspected of low quality. These are the flag for poor geophysical retrieval Fg_ctrl_poor_geophysical (which detects sun glint, galactic glint, wind speed and suspected ice), the flag for poor retrieval Fg_ctrl_poor_retrieval (bad convergence of the algorithm calculating SSS) and a quality flag specific for the roughness model used (Dg_quality_SSS1). A pixel with the three flags activated is removed from the dataset. While this quality flag setup is likely to allow pixels with a bad quality to be retained, the aim of this work is to filter these bad data in later steps with the DINEOF processing.

Two additional steps are performed in order to remove data with low quality. The first one consists of a range check in which pixels with a value too high or too low with respect to the monthly climatology calculated using the data described in Section 2.2 are removed. This test is performed on a pixel basis, and the difference threshold is set to \pm 2 . Finally, the outlier detection procedure described in Section 3.3, based on DINEOF, is applied. The parameters used for the outlier detection have been determined through a sensitivity analysis which will be shown in Section 4.1.

The final dataset has an average amount of missing data of $75.45 \%$, and the average percentage of missing data in space and time is shown in Fig. 1. The highest amount of missing data (more than $90 \%$ ) is found in the eastern part of the North Atlantic Ocean and the
Mediterranean Sea. Part of the eastern Mediterranean Sea does not contain any data after the quality checks (white areas in Fig. 1), and therefore these zones are not used in the rest of this work. The percentage of missing data is the lowest in September, and while it is difficult to determine the reason for this decrease in the percentage of missing data, a possible cause is that during this period of the year both temperature and salinity reach their highest values in the zone of study, which might result in a lower signal-to-noise ratio in the calculation of SSS, and therefore to more data passing the quality checks.

\subsection{DINEOF}

DINEOF (Data Empirical Orthogonal Functions, Beckers and Rixen (2003), Alvera-Azcárate et al. (2005)) has been used in this study in order to calculate daily SSS fields with low noise and reduced error. DINEOF consists of an EOF-based reconstruction of the missing data in a geophysical dataset, extracting the main patterns of variability from the data. It uses a truncated EOF basis to infer the missing data, and therefore noise can be effectively reduced in the reconstructed dataset, as noise is typically found in the higher order EOFs. Some transient information, however, can be also removed from the final result as these might be also found in the higher order EOFs. In order to calculate the EOF basis from a dataset that has missing data, the following steps are performed: first the monthly climatology described in Section 2.2 (interpolated to daily values) is removed from the data, and the missing values are set to zero (i.e. the mean of the dataset). A first EOF decomposition using only the first EOF is performed, and this reduced EOF basis is used to infer an improved estimation of the missing data. Once convergence has been reached for the estimation of the missing data using the first EOF, the whole procedure is repeated using 2 EOFs. Then $3,4 \ldots n$ EOFs are calculated. The total number of EOFs to be calculated, $n$, is determined by cross-validation: a set of initially valid data (about $3 \%$ of the total data) is set aside at the beginning and treated as missing data. At each step, a cross-validation error is calculated. The number of EOFs that minimizes this error is used as the optimal for the dataset reconstruction. More details about DINEOF and examples can be found in Beckers and Rixen (2003), Alvera-Azcárate et al. (2007, 2005).

A filter was applied to the temporal covariance matrix, based in Alvera-Azcárate, Barth, Sirjacobs, and Beckers (2009). This filter was initially implemented to improve the temporal sequence of the analyzed images. In case of large amounts of missing data (Alvera-Azcárate et al., 2009), or highly irregular time steps (Alvera-Azcárate et al., 2015), the filter allows improving the reconstruction by taking into account the temporal coherency of the data. In this case, the aim was to smooth the reconstruction and improve the temporal coherence of the data, as the percentage of missing data is very high in certain zones, which results in not enough data to perform a DINEOF reconstruction if no filter is used. In order to test the effect of this filter in the final quality of the data, two filter lengths are presented in the sensitivity study of Section 4.1.

\subsection{Outlier detection}

In order to detect outliers in the initial SSS dataset, before performing the final analysis using DINEOF, an outlier detection procedure consisting of three tests is applied to the data, following AlveraAzcárate, Sirjacobs, Barth, and Beckers (2012). In the first test ("EOF test") suspect data are detected as those presenting a large residual with respect to a truncated EOF basis calculated using DINEOF. The second test ("Median test") aims to detect departures from a local median (calculated in a 20 by 20 pixel box) and the third test ("Proximity test") penalizes more strongly pixels in the vicinity of land or missing data (e.g. at the edges of the swath, or near other pixels already classified as bad by the quality flags). A weighted combination of these three tests is performed (in this study, an equal weight of $1 / 3$ is given to all tests), and finally a threshold to classify a given pixel as an outlier is 
decided. Fig. 3 shows an example of the three outlier detection sub-tests for 7 February 2013. The effect of the final threshold in the quantity of eliminated data is shown in Fig. 4 for 7 February 2013 as well, where two thresholds are shown: 1 (stronger, i.e. more data are classified as outliers) and 2 (weaker). As expected, a higher concentration of outlier data is found at the edges of the swaths, near land and in the Mediterranean Sea. The initial SSS field and the resulting fields when applying the outlier detection with the two mentioned thresholds are shown in Fig. 5. The weights given to each sub-test and the final threshold depend on the initial data quality and the final use of the data. In this work the final threshold is set to one, which eliminates $12.14 \%$ of data (see Table 1 to see the impact of the outlier threshold in the amount of missing data). For more details and examples using other variables (sea surface temperature and chlorophyll-a concentration) the readers are referred to Alvera-Azcárate et al. (2012).

\section{Results}

\subsection{Sensitivity study}

Once the SSS data have been checked for their quality and the outliers have been removed, a DINEOF analysis is performed in order to obtain full SSS fields at daily temporal resolution and $0.15^{\circ} \times 0.15^{\circ}$ spatial resolution. In order to determine the optimal values for the temporal covariance filter and the outlier threshold, a series of analyses have been realized with varying values for these parameters. Regarding the length of the temporal covariance filter, two values are shown here: 3 and 14 days, representing a short and a long filter. Other tests were done with intermediate values that are not shown here. The 14-day filter, as shown in the following, provided better results than any of the other tested filter lengths. The repeat cycle of SMOS is 18 days, but, as addressed later in Section 4.5, using a 18-day length for the temporal covariance filter results in part of the signal considered erroneous being retained in the EOF basis, and therefore we chose 14 days as the maximum filter length in this work. The reader is cautioned however that the optimal filter length will vary from one dataset to another, and therefore an optimisation of this value must be realized for each dataset. A summary of the number of EOFs retained by the DINEOF
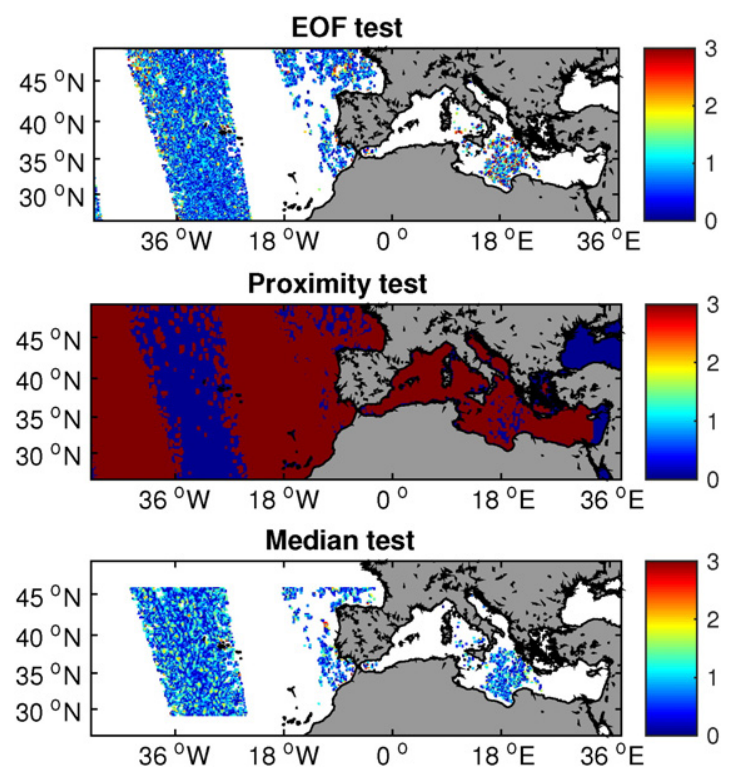

Fig. 3. Example of the three sub-tests for the detection of outliers for 7 February 2013. Top panel: EOF test (unitless, larger values mean larger departures from the EOF basis). Middle panel: proximity test (unitless; a value of 3 is assigned to pixels near land or missing data, zero value for the rest). Bottom panel: median test (unitless, larger values mean larger departures from the local median).
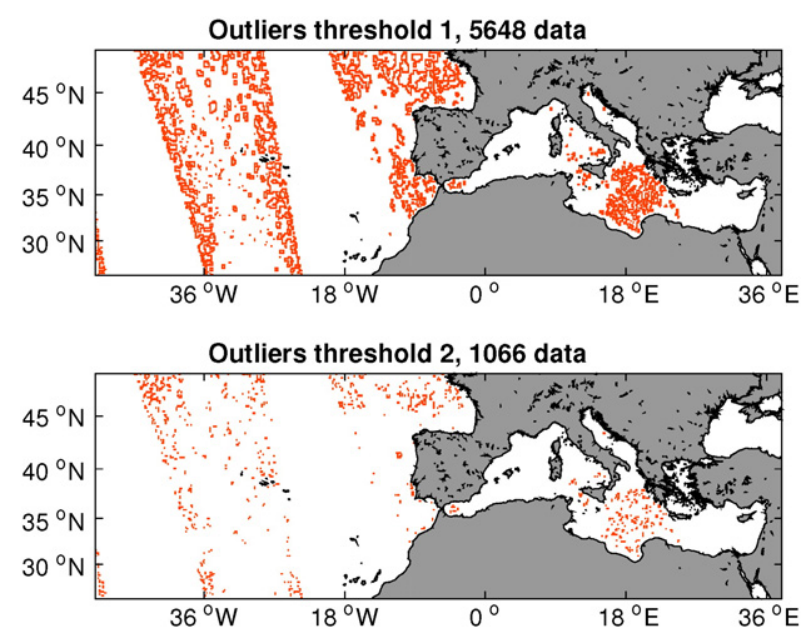

Fig. 4. Example of the data classified as outliers for 7 February 2013, using two thresholds 1 (top panel) and 2 (bottom panel). The total number of outliers detected for each threshold is given in the title of each sub-figure.

reconstruction, the variance explained and the cross-validation error obtained is included in Table 2.

Using a very short temporal covariance filter length (3 days) results in a reconstruction using one EOF, while the three reconstructions tested using a longer filter (14 days) results in reconstructions using 5 EOFs. The number of EOFs has an effect in the quantity of explained variance, and therefore in the quality of the final reconstruction. For this case, it therefore appears that longer filter lengths provide better results, with the reconstruction using an outlier threshold of 1 retaining the highest variance (78\%, see Table 2 ).

In order to establish the impact of the different parameters used, a comparison of each reconstruction with the in situ validation dataset and the TSG data described in Section 2.2 has been realized. The results of this comparison are summarized in Table 3. It can be seen that, in general, the choice of the outlier threshold level has a strong influence in the quality of the final result: for example, the centered RMS error of the DINEOF reconstructed data when compared with TSG data is of 0.6 for a filter length of 14 days and outlier threshold of 2 , and this value decreases to 0.55 when the outlier threshold is 1 . For a filter length
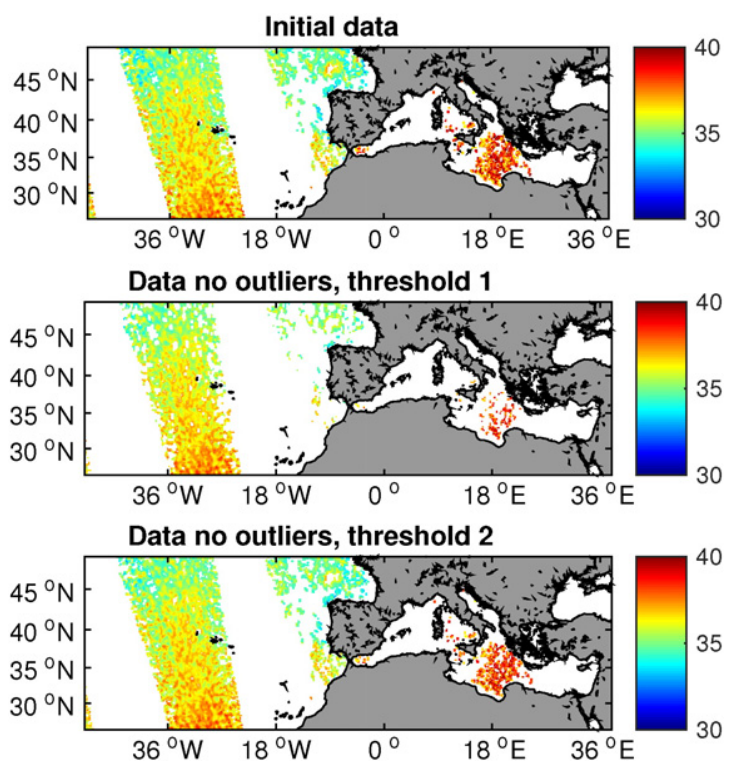

Fig. 5. Top panel: initial SSS data (after quality checks) for 7 February 2013. Middle panel: initial data with outliers removed, using a threshold of 1 . Bottom panel: initial data with outliers removed, using a threshold of 2 . 
Table 1

Percentage of missing data in the initial SMOS SSS dataset (after the quality checks described in Section 2.1) and after the outlier detection tests, using 3 thresholds.

\begin{tabular}{ll}
\hline Dataset & Missing data \\
\hline Initial data (after QC checks) & $63.31 \%$ \\
Initial data with outlier threshold =2.0 & $64.87 \%$ \\
Initial data with threshold $=1.5$ & $68.84 \%$ \\
Initial data with threshold $=1.0$ & $75.45 \%$ \\
\hline
\end{tabular}

of 3 days and an outlier threshold of 1, the centered RMS decreases further to 0.49. Similar reductions are observed in the comparison with WOD and Coriolis data, although the number of match-ups is very low. These results lead to the conclusion that an outlier threshold of 1 is the best choice. Regarding the filter length, it appears that the short filter value (i.e. 3 days) leads to improved results, although as it has been shown in Table 2, this reconstruction uses only one EOF, which decreases the explained variance and results in a smoother reconstruction which may explain the lower error values. Large fresh biases are observed in all comparisons. As mentioned before, DINEOF is not able to remove constant or persistent biases from the initial data. As a conclusion of the sensitivity study, the results of the reconstruction using a filter length of 14 days and an outlier threshold of 1 will be used in the remaining of this work.

\subsection{Comparison with TSG data}

The comparison with TSG data in Table 3 reveals that the fresh bias observed in the initial data $(-0.39)$ increases to -0.57 in the reconstruction provided by DINEOF (Table 3). In order to assess the causes of this increase, the comparison between TSG and SMOS data will be looked at with more detail. Fig. 6 shows the TSG data and the SMOS data (anomaly with respect to the TSG data) at the TSG positions. The DINEOF data along the French, Spanish and Portuguese coasts present the lowest values with respect to the TSG data. These data are not present in the original SMOS data, and therefore the bias of the original SMOS data appears -artificially- to be lower than the DINEOF reconstruction. Large biases in SMOS SSS can be expected near land due to land contamination issues. Latitudinal or large scale bias is also present in the original data, and as mentioned before DINEOF does not aim at correcting these sources of bias. Fig. 6 also includes the DINEOF estimate at the initially present SMOS positions, showing the ability of DINEOF to reduce noise in the data.

Fig. 7 shows a scatter plot comparing the initial SMOS data and DINEOF data (at positions initially present in SMOS and at all points), with the color representing the longitude of the data. It can be seen that the DINEOF results at positions initially present in SMOS are much closer to the 1:1 line than the original SMOS data. When looking at the DINEOF results at all points we can see that data at around $10^{\circ} \mathrm{W}$ depart largely from the $1: 1$ line, and these are again the data near the French, Spanish and Portuguese coasts. The DINEOF SSS data at the Mediterranean Sea (in deep red in Fig. 7, longitudes larger than $0^{\circ} \mathrm{E}$ ) do present also a slightly larger bias than the average data, but this bias is smaller than the one along the French, Spanish and Portuguese coasts in the North Atlantic Ocean.

Table 2

Number of EOFs retained by DINEOF, percentage of explained variance and cross-validation error for analyses using different values of the covariance filter length and outlier threshold.

\begin{tabular}{lllll}
\hline Filter length & Outlier threshold & \# EOFs & $\begin{array}{l}\text { Variance } \\
\text { explained }\end{array}$ & $\begin{array}{l}\text { Cross-validation } \\
\text { error }\end{array}$ \\
\hline 3 & 1 & 1 & $64 \%$ & 0.76 \\
14 & 1 & 5 & $78 \%$ & 0.75 \\
14 & 1.5 & 5 & $71 \%$ & 0.76 \\
14 & 2 & 5 & $68 \%$ & 0.75 \\
\hline
\end{tabular}

\section{Table 3}

Comparison statistics between in situ data and SMOS data for two filter lengths ( 3 days and 14 days) and two outlier threshold levels (1.0 and 1.5). The two in situ datasets used (WOD/Coriolis and thermosalinograph data) are described in the text. The DINEOF reconstruction is compared at initially present points and at all available points. Statistics used are Root Mean Square Error (RMS), centered RMS (CRMS), bias and Correlation (r). The number of data used for each calculation is included. Note the low number of data available for some of the comparisons using initial SMOS data.

\begin{tabular}{|c|c|c|c|c|c|}
\hline & RMS & CRMS & Bias & $\mathrm{r}$ & \# data \\
\hline \multicolumn{6}{|l|}{ Filter length 3 days } \\
\hline \multicolumn{6}{|l|}{ Outlier threshold 1.0} \\
\hline WOD/Coriolis initial & 0.85 & 0.76 & -0.37 & 0.33 & 11 \\
\hline WOD/Coriolis DINEOF initial points & 0.36 & 0.31 & -0.18 & 0.82 & 11 \\
\hline WOD/Coriolis DINEOF & 0.55 & 0.54 & -0.1 & 0.79 & 1557 \\
\hline TSG initial & 0.67 & 0.55 & -0.4 & 0.79 & 29 \\
\hline TSG DINEOF initial points & 0.42 & 0.33 & -0.26 & 0.89 & 29 \\
\hline TSG DINEOF & 0.71 & 0.49 & -0.52 & 0.88 & 184 \\
\hline \multicolumn{6}{|l|}{ Filter length 14 days } \\
\hline \multicolumn{6}{|l|}{ Outlier threshold 1.0} \\
\hline WOD/Coriolis initial & 0.71 & 0.7 & -0.1 & 0.7 & 8 \\
\hline WOD/Coriolis DINEOF initial points & 0.43 & 0.41 & -0.14 & 0.76 & 8 \\
\hline WOD/Coriolis DINEOF & 0.67 & 0.64 & -0.2 & 0.72 & 1557 \\
\hline TSG initial & 0.67 & 0.55 & -0.39 & 0.79 & 29 \\
\hline TSG DINEOF initial points & 0.53 & 0.37 & -0.38 & 0.87 & 29 \\
\hline TSG DINEOF & 0.79 & 0.55 & -0.57 & 0.86 & 184 \\
\hline \multicolumn{6}{|l|}{ Outlier threshold 1.5} \\
\hline WOD/Coriolis initial & 0.64 & 0.6 & -0.24 & 0.5 & 26 \\
\hline WOD/Coriolis DINEOF initial points & 0.52 & 0.49 & -0.17 & 0.78 & 26 \\
\hline WOD/Coriolis DINEOF & 0.68 & 0.64 & -0.2 & 0.72 & 1567 \\
\hline TSG initial & 0.73 & 0.6 & -0.43 & 0.77 & 38 \\
\hline TSG DINEOF initial points & 0.56 & 0.35 & -0.4 & 0.92 & 38 \\
\hline TSG DINEOF & 0.86 & 0.57 & -0.64 & 0.85 & 184 \\
\hline \multicolumn{6}{|l|}{ Outlier threshold 2} \\
\hline WOD/Coriolis initial & 0.77 & 0.67 & -0.37 & 0.67 & 49 \\
\hline WOD/Coriolis DINEOF initial points & 0.61 & 0.58 & -0.19 & 0.56 & 49 \\
\hline WOD/Coriolis DINEOF & 0.72 & 0.67 & -0.27 & 0.7 & 1568 \\
\hline TSG initial & 0.87 & 0.70 & -0.51 & 0.73 & 44 \\
\hline TSG DINEOF initial points & 0.61 & 0.4 & -0.47 & 0.90 & 44 \\
\hline TSG DINEOF & 0.92 & 0.6 & -0.7 & 0.84 & 184 \\
\hline
\end{tabular}

It should also be noted that the error statistics of Table 3 include zones that are very close to land and the Mediterranean Sea, which are typically not available in other SMOS SSS products, and therefore not used in comparisons with in situ data. A comparison using only DINEOF SSS data at open ocean locations (west of $24^{\circ} \mathrm{W}$ ) reveals a centered RMS of 0.61 when comparing to WOD and Coriolis data and of 0.32 when comparing to TSG data (Table 4), a reduction of $40 \%$ with respect to the error of the initial SMOS data.

\subsection{Spatial resolution of the reconstruction}

In order to show the effective spatial resolution that the DINEOF reconstruction is able to retain, two transects of TSG data are compared to the SMOS data at very high spatial resolution. Co-located transects of TSG salinity, SMOS and DINEOF SSS are shown in Fig. 8. The TSG data

\section{Table 4}

Comparison statistics between in situ data and SMOS data for open waters (longitudes west of $24^{\circ} \mathrm{W}$ ), for a filter length of 14 days and an outlier threshold of 1 . There are not enough match-up points to assess the error between SMOS data and WOD. Statistics used are Root Mean Square Error (RMS), centered RMS (CRMS), bias and Correlation ( $r$ ). The number of data used for each calculation is included. Note the low number of data available for some of the comparisons using initial SMOS data.

\begin{tabular}{llllll}
\hline & RMS & CRMS & Bias & r & \# data \\
\hline WOD/Coriolis initial & - & - & - & - & - \\
WOD/Coriolis DINEOF initial points & - & - & - & - & - \\
WOD/Coriolis DINEOF & 0.62 & 0.61 & -0.12 & 0.67 & 1372 \\
TSG initial & 0.59 & 0.53 & -0.25 & 0.66 & 20 \\
TSG DINEOF initial points & 0.28 & 0.19 & -0.2 & 0.91 & 20 \\
TSG DINEOF & 0.39 & 0.32 & -0.22 & 0.94 & 48
\end{tabular}



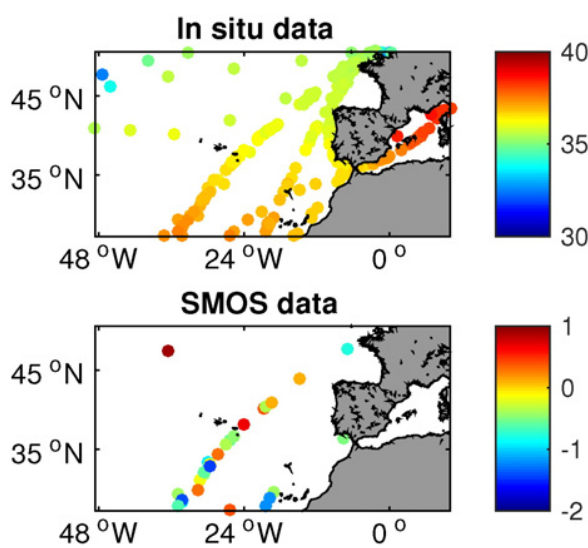

DINEOF (SMOS positions)
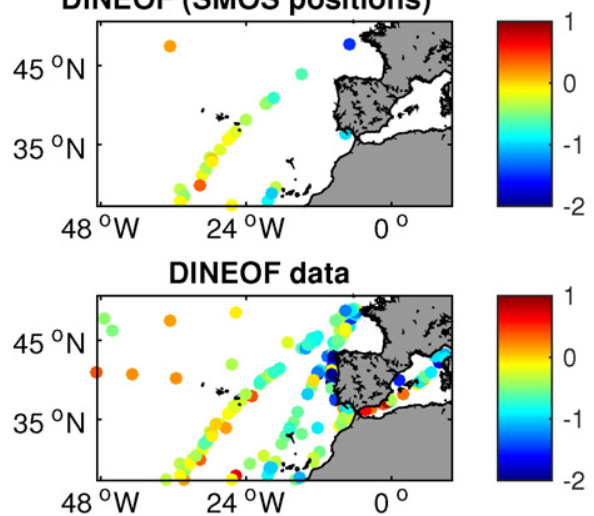

Fig. 6. First panel: TSG data, averaged daily. Second panel: original SMOS SSS data anomalies with respect to the TSG data. Third panel: DINEOF reconstruction of SSS data (anomalies with respect to the TSG data) at the initially present SMOS positions. Fourth panel: DINEOF reconstruction of SSS data anomalies with respect to the TSG data at all positions. Fresh biases can be seen along the French, Spanish and Portuguese coasts.

are kept at their original temporal resolution $(\sim 5 \mathrm{~min})$ in order to show the capability of the DINEOF SSS to reproduce small-scale variations. The original and DINEOF SSS data (daily data at $0.15^{\circ} \times 0.15^{\circ}$ ) are interpolated to the TSG positions. Two transects are shown, one getting closer to the European coasts. It is observed that DINEOF is able to strongly reduce the noise in SMOS data, and at the same time reproduce the small-scale variations observed in the TSG data. There is a constant fresh bias in the DINEOF data, that increases when getting close to the coast (east of $24^{\circ} \mathrm{W}$ ). The centered RMS for transect $\mathbf{a}$ is 0.22 and the bias is -0.35 . For transect $\mathbf{b}$, which is located only in open ocean, the centered RMS is 0.2 and the bias is -0.3 . These results show the capability of DINEOF to retain the high resolution of the initial dataset.

\subsection{Reconstruction results}

An example of the reconstructed SSS provided by DINEOF is shown in Fig. 9 for 21 July 2013. This example shows the capability of DINEOF to reduce the noise still present in the daily SSS fields after the quality checks and the outlier check performed in this work. The Gulf Stream is well observed in the top left part of the domain, with fresher waters and meanders of about $200 \mathrm{~km}$ in size. Two low salinity zones around the Gironde (France) and the Douro (Portugal) rivers are also observed, indicating that these river plumes can as well have a signature in the SMOS SSS maps, once the appropriate corrections have been applied. Large river plumes have already been studied using SMOS data (e.g. the Amazon river plume, Grodsky et al., 2014; Reul et al., 2014; Fournier et al., 2015), but the Douro and Gironde river plumes, because of their smaller size and the large biases caused by land contamination, have not yet been assessed using SMOS data. Although it is difficult to assess quantitatively the accuracy of the SMOS data at these river
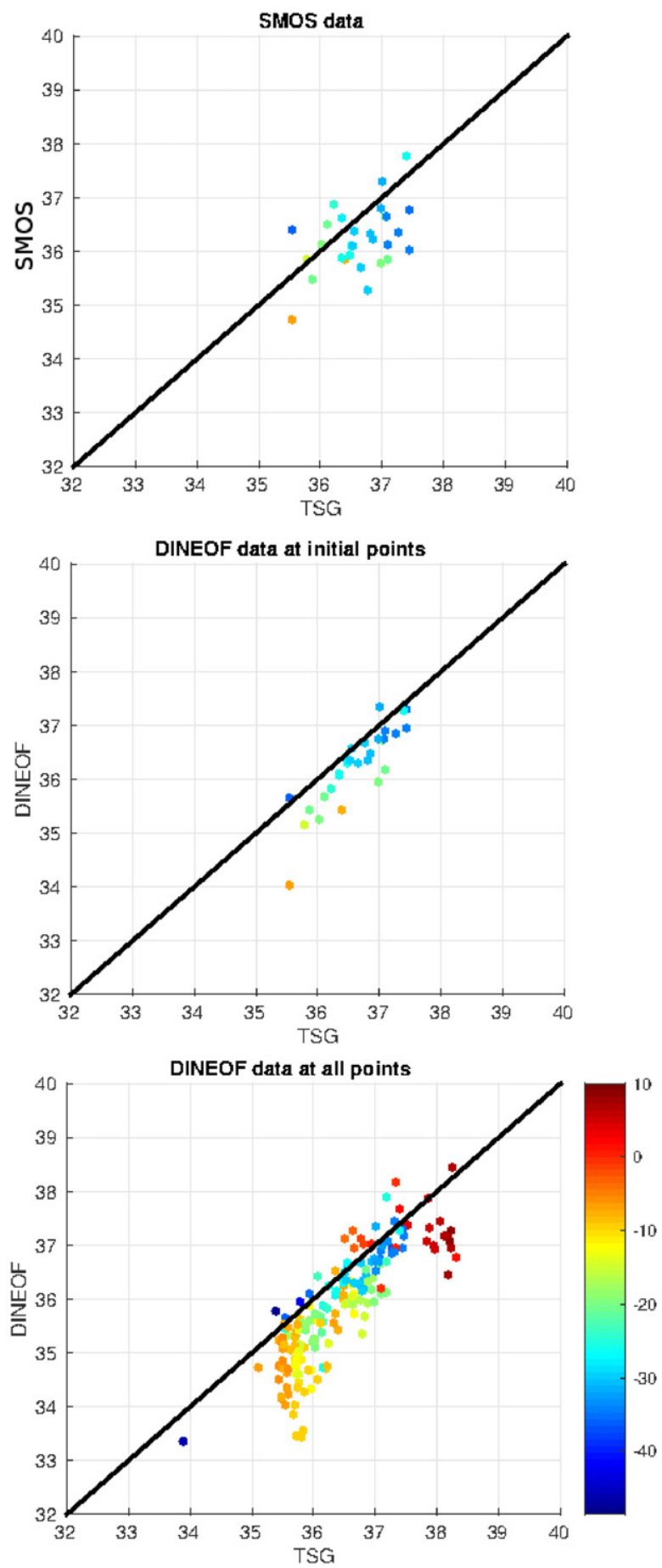

Fig. 7. Scatter plots comparing the SMOS data and DINEOF reconstruction to TSG data. Top panel: SMOS data vs. TSG data. Middle panel: DINEOF reconstruction at originally present points vs. TSG data. Bottom panel: DINEOF reconstruction at all points vs. TSG data. The colors indicate the longitude of each point in the graphic.

plumes, the qualitative description of these signals can be helpful to analyze the extent of the plumes and their seasonal variability.

Fig. 10 presents the averaged salinity time series at the Douro and Gironde river plumes along 2013 (the boxes delimiting these two zones are shown in Fig. 9). The Douro river plume shows values ranging from 33 to 35.4. The Gironde plume shows values ranging from 32.2 to 33.7, with minimum values in June and November 2013. Following Jalón-Rojas, Schmidt, and Sottolichio (2015), a flood event occurred in 


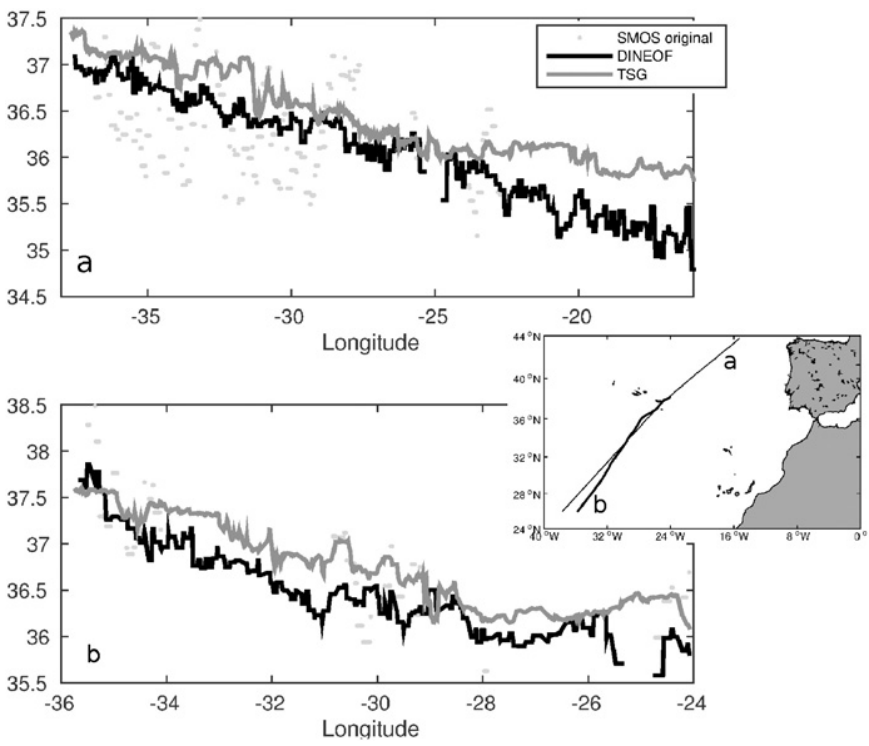

Fig. 8. Two TSG transects at their original temporal resolution $(\sim 5 \mathrm{~min})$ in the North Atlantic Ocean and their SMOS and DINEOF interpolated to the TSG positions. Transect a (top panel) gets closer to the coast and this is reflected in the larger fresh bias in the DINEOF data.

the Gironde estuary in June 2013 (see their Fig. 3), which explains the low salinity values observed in the DINEOF reconstruction. This minimum in the DINEOF SSS is observed to develop quickly at the beginning of June, and erode during July (data not shown). The minimum SSS observed in November in Fig. 10 can also be correlated to a peak in river discharge (Fig. 3 in Jalón-Rojas et al., 2015).

While part of the low salinity signal observed at the Portuguese coast is certainly due to land contamination, the lowest SSS amplitudes are found near the mouth of the Douro river. By inspecting the initial SSS data (after check for outliers), and the DINEOF reconstruction, averaged over the whole 2013, a zone of lower SSS is observed in the vicinity of the Douro river mouth, extending about $200 \mathrm{~km}$ from the coast. This signal is also observed in the background field described in Section 2.2, which is independent of the original SMOS SSS data. In order to investigate if this signal could be produced by the presence of fresh water from the Douro river, the chlorophyll-a data described in Section 2.1 were analyzed. Mendes et al. (2014) describe a circular signature in MODIS data due to the presence of the plume, which varies in form and expansion from the coast due to the wind regime. The plume of the Douro is visible in the chlorophyll-a data at several instances through 2013, with varying magnitude, and the location and size corresponds to what is observed in SSS. An example for the period from 26 February to 5 March is shown in Fig. 11. It can be seen that the zone of the lowest SSS near

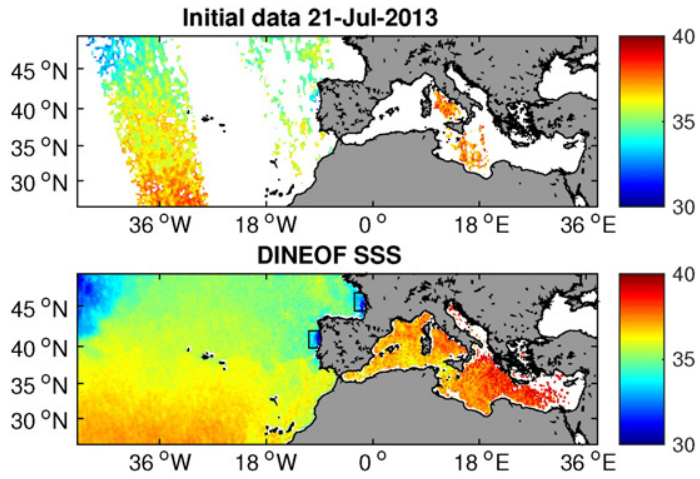

Fig. 9. Example of initial (top panel) and reconstructed DINEOF SSS data (bottom panel) for 21 July 2013
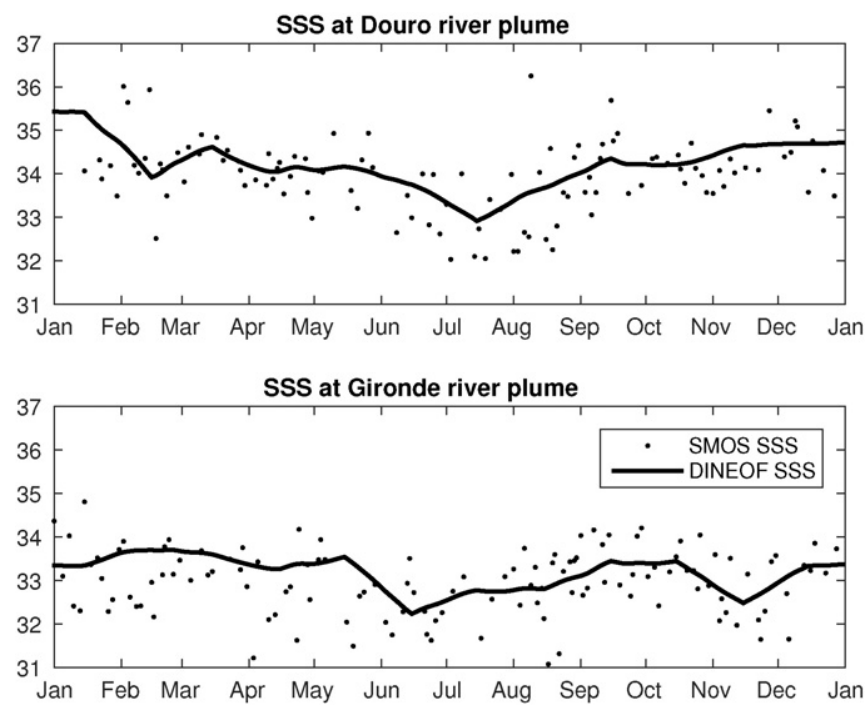

Fig. 10. Time series of SSS at the Douro (top panel) and the Gironde (bottom panel) river plumes. The boxes delimiting these two zones are shown in Fig. 9.

the mouth of the river is associated with a higher chlorophyll-a concentration, and that the plume has a spatial signature that reaches a longitude of $\sim 11^{\circ} \mathrm{W}$. The fact that the plume is not detected in the TSG data shown in Fig. 6 can be due to the fact that the TSGs are often located at depths ranging from 6 to $10 \mathrm{~m}$, while the Douro plume might be shallower than $10 \mathrm{~m}$ (Iglesias, Couvelard, Avilez-Valente, \& Caldeira, submitted for publication).

The Mediterranean SSS reconstruction provided by DINEOF presents a large bias with respect to TSG data (Fig. 7), but as can be seen in Fig. 9 the DINEOF data are able to reproduce the east-west salinity gradient observed in the Mediterranean Sea (e.g. Brasseur, Beckers, Brankart, \& Schoenauen, 1996). The DINEOF SSS average value is 35.5 for the western Mediterranean basin and 38 in the eastern Mediterranean basin when considering the whole 2013 dataset. For the particular day shown in Fig. 9, 21 July 2013, the average SSS value is 37 in the western Mediterranean basin and 38 in the eastern Mediterranean basin. Therefore, while a careful bias correction procedure should be applied, this example shows the approach used in this work allows obtaining a first estimate of the SSS in this zone.

\subsection{EOF basis}

The DINEOF reconstruction used in this work consists of 5 EOF modes that explain $78 \%$ of the initial variance (Table 2). The five spatial EOFs are shown in Fig. 12 and the temporal ones in Fig. 13. These show the spatial and temporal evolution of the Gulf Stream and its meanders,
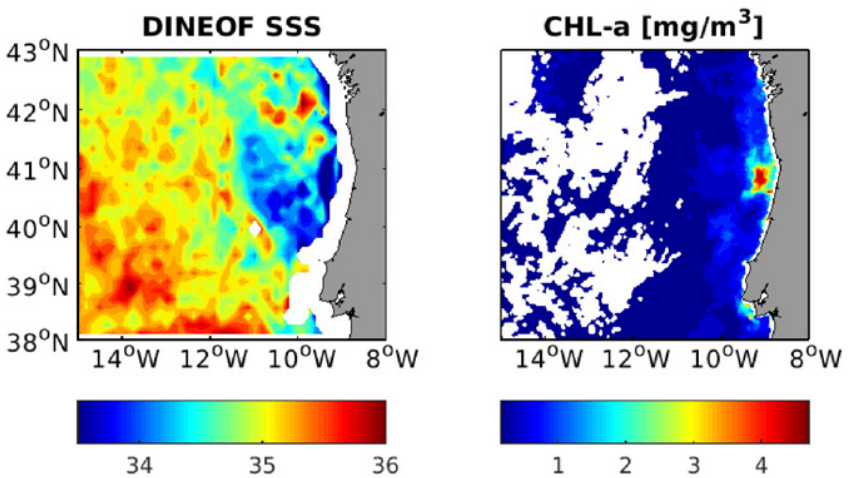

Fig. 11. Example of the signal of the Douro river plume in SSS (left panel) and chlorophylla concentration (right panel) averaged over the period 26 February to 5 March 2013. 
and the subtropical SSS maximum. Other features that appear to have a signal in the EOF basis are the Alboran Sea (EOF 1) that becomes saltier through the year, and the plumes of the Gironde (EOF 1 ) and Douro rivers (EOFs 1 and 3). An in-depth study of the signature of these three features in the SMOS SSS needs to be carried on in future works.

Two of the EOFs rejected by DINEOF (i.e. not used in the final reconstruction) present a pattern that is easily attributed to the errors at the edges of the satellite swath (Fig. 14). These patterns, with a periodicity of 18 days, represent about $2.8 \%$ of the initial variance and have been isolated by the EOF decomposition. Because these EOF modes are not retained in the EOF basis used for the DINEOF reconstruction, the errors at the edges of the swath are effectively removed from the dataset. This approach allows retaining more information in the initial dataset while removing constant errors. The length of the temporal covariance filter has an effect in the detection of these patterns: when using a filter of a length of 18 days, these features were not captured in any EOF mode (results not shown), indicating that the signal was being averaged within the data. By selecting a shorter filter length we were able to smooth the data in time but at the same time to isolate and remove these features from the data, which validates the choice of a 14-day filter length.

\section{Conclusions}

A procedure to obtain SMOS SSS data at a daily time step and with a spatial resolution of $0.15^{\circ} \times 0.15^{\circ}$ using DINEOF has been presented. It
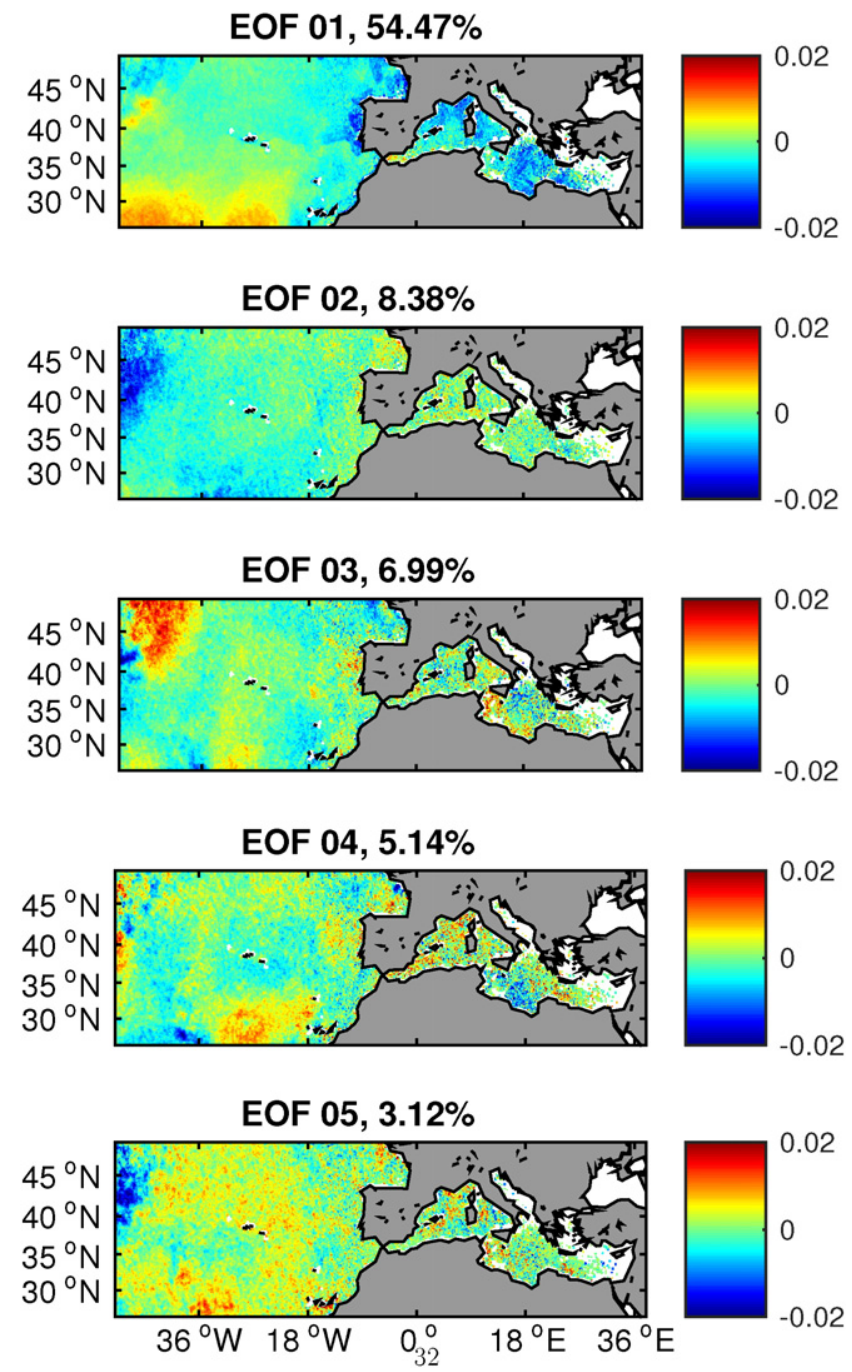

Fig. 12. Five EOF spatial modes retained for the DINEOF reconstruction. The percentage in each title provides the explained variance.

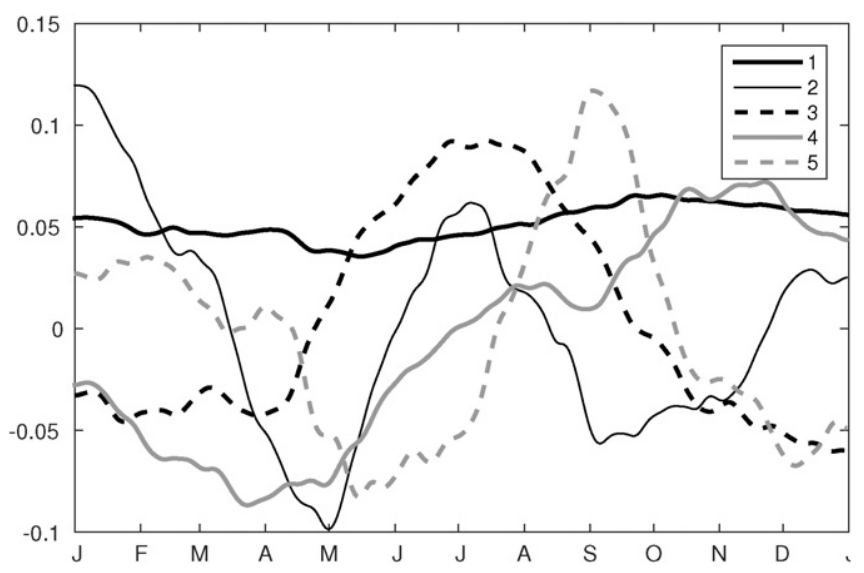

Fig. 13. Five EOF temporal modes retained for the DINEOF reconstruction.

has been shown that DINEOF allows retrieving complete daily fields of SSS with reduced noise, by detecting and removing outliers in the initial fields. A sensitivity study has been carried out to determine the optimal configuration of DINEOF, namely the length of a temporal covariance filter (aiming at improving the temporal coherence of the dataset) and the outlier detection threshold. The truncated EOF basis used to compute the final SSS data is able to reject noise, as well as errors present at the edges of the satellite swath. Comparison with in situ data shows that in addition to the increased coverage, the correlation is improved and the centered RMS error is reduced. The centered RMS error between the DINEOF SSS estimates (at the highest spatial and temporal resolution) and TSG data in open waters is as low as 0.2. When considering the whole domain of study (including the Mediterranean Sea) the centered RMS increases to 0.55 . Constant biases present in the initial dataset cannot be corrected using DINEOF, as these persistent signals are probably in the most dominant EOFs, and therefore affect the final dataset as well. The bias has been shown to be of -0.3 in open waters (in comparisons with TSG data) and -0.57 over the whole domain of study.

The presence of several features in the reconstructed dataset has been assessed: the Gulf Stream and the subtropical salinity maximum at the large spatial scale, and the fresh plumes of the Gironde and Douro rivers at the small scale. The presence of the river plumes in the SMOS data is evidenced by a localized salinity minimum that is superimposed to constant biases present along the coast and that are due to the presence of land masses. Because of these biases, the study of these plumes cannot be done quantitatively, but the DINEOF SSS data allow assessing their spatial extent and variation with time. The time series of SSS at the vicinity of the Gironde estuary correlates well to the river discharge, with the minimum SSS occurring during a flood event in June 2013. Regarding the Douro river plume, it has been shown that it presents a shape and size similar to what is observed in chlorophyll-a concentration data. A careful validation of these results as well as an assessment of the spatial and temporal scales of these river plumes must be carried out using other sources of data (satellite and in situ) and hydrodynamic models.

While errors still remain in the DINEOF reconstruction, these can be further reduced through bias correction procedures and through spatial and temporal averaging of the data, as for example the one proposed by Kolodziejczyk et al. (2016-in this issue). The aim of this work was to provide SSS data at the highest resolution possible in space and time in order to remove errors at their source. Future work includes the multivariate analysis of SSS with variables like temperature and precipitation, in order to establish the correlation between them and use this information in the improvement of the SSS estimations. The assessment of the spatial and temporal variability of the river plumes observed in this work, and their correlation with river discharge and turbidity will be also the focus of future studies. 
Spatial EOF 06, Explained variance $1.42 \%$

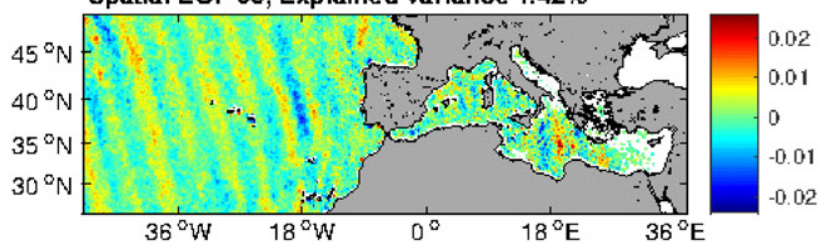

Temporal EOF 06

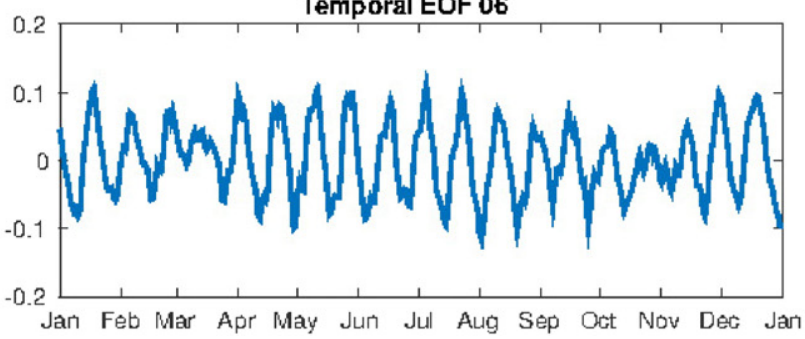

Spatial EOF 07, Explained variance $1.41 \%$

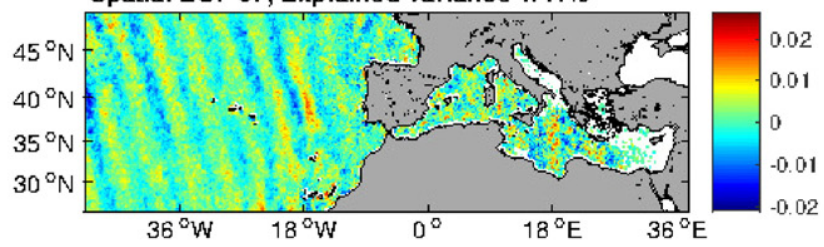

Temporal EOF 07

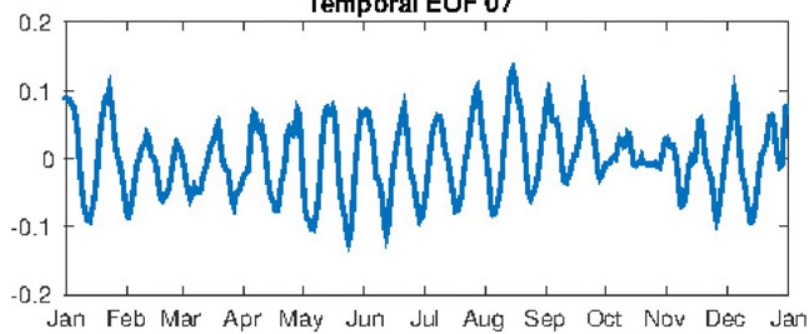

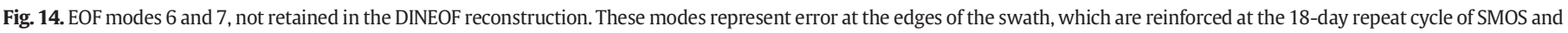
therefore isolated as two distinct EOF modes. Note that the temporal EOFs have a quarter-phase lag between them.

\section{Acknowledgments}

This work has been carried out within the project "Improving Sea Surface Salinity Estimates through Multivariate and Multisensor Analyses" (http://www.gher.ulg.ac.be/WP/), funded by the European Space Agency (4000110775/14/I-BG) "Support to Science (STSE) PATHFINDERS" call.

In situ data providers, Coriolis Data Centre (http://www.coriolis.eu. org) and World Ocean Database (http://www.nodc.noaa.gov/) are also acknowledged. Sea surface salinity data derived from thermosalinograph instruments installed onboard voluntary observing ships were collected, validated, archived, and made freely available by the French Sea Surface Salinity Observation Service (http://www.legos.obs-mip.fr/ observations/sss/). Chlorophyll-a concentration data were obtained through http://oceancolor.gsfc.nasa.gov/. We also thank Manuel Arias for his feedback on the use of SMOS salinity quality flags.

\section{References}

Alvera-Azcárate, A., Barth, A., Beckers, J. -M., \& Weisberg, R. H. (2007). Multivariate reconstruction of missing data in sea surface temperature, chlorophyll and wind satellite fields. Journal of Geophysical Research, 112, C03008. http://dx.doi.org/10.1029/ 2006JC003660.

Alvera-Azcárate, A., Barth, A., Rixen, M., \& Beckers, J. -M. (2005). Reconstruction of incomplete oceanographic data sets using Empirical Orthogonal Functions. Application to the Adriatic Sea surface temperature. Ocean Modelling, 9, 325-346. http://dx.doi. org/10.1016/j.ocemod.2004.08.001.

Alvera-Azcárate, A., Barth, A., Sirjacobs, D., \& Beckers, J. -M. (2009). EOF expansions for the reconstruction of missing data using DINEOF. Ocean Science, 5, 475-485.

Alvera-Azcárate, A., Sirjacobs, D., Barth, A., \& Beckers, J. -M. (2012). Outlier detection in satellite data using spatial coherence. Remote Sensing of Environment, 119, 84-91.

Alvera-Azcárate, A., Vanhellemont, O., Ruddick, K., Barth, A., \& Beckers, J. -M. (2015). Analysis of high frequency geostationary ocean colour data using DINEOF. Estuarine, Coastal and Shelf Science, 159, 28-36.

Barth, A., Beckers, J. -M., Troupin, C., Alvera-Azcárate, A., \& Vandenbulcke, L. (2014). divand-1.0: $n$-dimensional variational data analysis for ocean observations. Geoscientific Model Development. 7. (pp. 225-241), 225-241 (URL http://www.geosci-model-dev. net $/ 7 / 225 / 2014 /$ ).

Beckers, J. -M., \& Rixen, M. (2003). EOF calculations and data filling from incomplete oceanographic data sets. Journal of Atmospheric and Oceanic Technology, 20(12), 1839-1856.

Boutin, J., Martin, N., Reverdin, G., Morisset, S., Yin, X., Centurioni, L., \& Reul, N. (2014). Sea surface salinity under rain cells: SMOS satellite and in situ drifters observations. Journal of Geophysical Research, Oceans, 119, 5533-5545.

Boutin, J., Martin, N., Xiaobin, Y., Font, J., Reul, N., \& Spurgeon, P. (2012). First assessment of SMOS data over open ocean: Part II - Sea surface salinity. IEEE Transactions on Geoscience and Remote Sensing, 50(5-1), 1662-1675 (50 (5), 1662-1675).
Boyer, T., Antonov, J. I., Baranova, O. K., Coleman, C., Garcia, H. E., Grodsky, A., ... Zweng, M. M. (2013). World Ocean Database 2013, NOAA Atlas NESDIS 72. In S. levitus (Ed.), a. mishonov, technical ed. Edition. MD: Silver Spring (209 pp., http://doi.org/10.7289/ V5NZ85MT).

Brasseur, P., Beckers, J. M., Brankart, J. M., \& Schoenauen, R. (1996). Seasonal temperature and salinity fields in the Mediterranean Sea: Climatological analyses of a historical data set. Deep-Sea Research Part I, 43, 159-192.

Fournier, S., Chapron, B., Salisbury, J., Vandemark, D., \& Reul, N. (2015). Comparison of spaceborne measurements of sea surface salinity and colored detrital matter in the amazon plume. Journal of Geophysical Research, Oceans, 120, 3177-3192.

Grodsky, S., Reverdin, G., Carton, J., \& Coles, V. (2014). Year-to-year salinity changes in the Amazon plume: contrasting 2011 and 2012 Aquarius/SACD and SMOS satellite data. Remote Sensing of Environment, 140, 14-22.

Iglesias, I., Couvelard, X., Avilez-Valente, P., \& Caldeira, R. (2016). Numerical study of the Douro River plume: Mediating the inner- and cross-shelf dynamics. Ocean Dynamics (submitted for publication).

Jalón-Rojas, I., Schmidt, S., \& Sottolichio, A. (2015). Turbidity in the fluvial gironde estuary (southwest france) based on 10-year continuous monitoring: Sensitivity to hydrological conditions. Hydrology and Earth System Sciences, 19, 2805-2819.

Kolodziejczyk, N., Boutin, J., Vergely, J. -L., Marchand, S., Martin, N., \& Reverdin, G. (2016). Mitigation of systematic errors in smos sea surface salinity. Remote Sensing of Environment, 180, 164-177 (in this issue).

Kolodziejczyk, N., Hernandez, O., Boutin, J., \& Reverdin, G. (2015). SMOS salinity in the subtropical North Atlantic salinity maximum: 2. Two-dimensional horizontal thermohaline variability. Journal of Geophysical Research. Oceans, 120, 972-987.

Lagerloef, G., \& Font, J. (2010). SMOS and aquarius/SAC-D missions: The era of spaceborne salinity measurements is about to begin. Springer (Ch. 3).

Lagerloef, G., Schmitt, R., Schanze, J., \& Kao, H. -Y. (2010). The ocean and the global water cycle. Oceanography, 23(4), 82-93.

Mendes, R., Vaz, N., Fernndez-Nvoa, D., da Silva, J., deCastro, M., Gmez-Gesteira, M., \& Dias, J. (2014). Observation of a turbid plume using MODIS imagery: The case of Douro estuary (Portugal). Remote Sensing of Environment, 154, 127-138.

Reul, N., Chapron, B., Lee, T., Donlon, C., Boutin, J., \& Alory, G. (2014). Sea surface salinity structure of the meandering Gulf Stream revealed by SMOS sensor. Geophysical Research Letters, 41, 3141-3148.

Sirjacobs, D., Alvera-Azcárate, A., Barth, A., Lacroix, G., Park, Y., Nechad, B., ... Beckers., J. -M. (2011). Cloud filling of ocean color and sea surface temperature remote sensing products over the Southern North Sea by the Data Interpolating Empirical Orthogonal Functions methodology. Journal of Sea Research, 65(1), 114-130.

Troupin, C., Barth, A., Sirjacobs, D., Ouberdous, M., Brankart, J. -M., Brasseur, P., ... Beckers, J. -M. (2012). Generation of analysis and consistent error fields using the data interpolating variational analysis (diva). Ocean Modelling (URL http://hdl.handle.net/ 2268/125731).

Yin, X., Boutin, J., Martin, N., \& Spurgeon, P. (2012). Optimization of L-band sea surface emissivity models deduced from SMOS data. IEEE Transactions on Geoscience and Remote Sensing, 50(5), 1414-1426.

Yin, X., Boutin, J., Martin, N., Spurgeon, P., Vergely, J. -L., \& Gaillard, F. (2014). Errors in SMOS Sea surface salinity and their dependency on a priori wind speed. Remote Sensing of Environment, 146, 159-171.

Zine, S., Boutin, J., Font, J., Reul, N., Waldteufel, P., Gabarró, C., ... Delwart, S. (2008). overview of the smos sea surface salinity prototype processor. IEEE Transactions on Geoscience and Remote Sensing, 46(3), 621-645. 